\title{
Impact of LOS/NLOS Transitions on Signal Quality Predictions
}

\author{
Caleb G. Braga, Victor F. Monteiro, Diego A. Sousa, Tarciso F. Maciel and Fco. Rodrigo P. Cavalcanti
}

\begin{abstract}
An envisioned characteristic of beyond $5 G$ networks is the regular use of Machine Learning (ML) based solutions in order to enhance the network performance. One possibility is to predict the signal quality in advance in order to anticipate actions, e.g., anticipate signaling related to handover and preallocate resources. One important point that must be investigated is how ML based models are impacted by dynamic changes in the environment. More specifically, the present work investigates the impact of LOS/NLOS transitions on signal quality prediction. The selected algorithm for this study was the well known ARIMA. Simulation results showed that the LOS/NLOS transition is a critical moment for predictions, since previous and future samples are highly uncorrelated. Besides, it was also shown that varying the prediction size window impacts more than varying the user speed.
\end{abstract}

Keywords-Channel prediction, ARIMA, 5G.

\section{INTRODUCTION}

The next generation of wireless telecommunications, the Fifth Generation (5G), is arriving to provide connectivity to a new range of services [1]. The $5 \mathrm{G}$ mainly focuses on three use cases: Enhanced Mobile Broadband (eMBB), Massive Machine-Type Communications (mMTC) and Ultra-Reliable Low-Latency Communications (URLLC). The eMBB addresses services that need high bit rates, e.g., $4 \mathrm{~K}$ video streaming. The mMTC enables the communication among a high number of machines, e.g., Internet of Things services such as smart metering. Finally, the URLLC focuses on services that require low latency and reliable communication, such as autonomous cars.

One of the novelties of $5 \mathrm{G}$ to meet the demands of these use cases is the utilization of millimeter Wave (mmWave) frequencies, e.g., $28 \mathrm{GHz}$. In mmWave frequencies there are still large portions of available spectrum. However, in that part of the spectrum the signal propagation loss is higher compared to lower frequencies. Besides, another challenge using the mmWave frequencies for communication is that the signals also suffer more to trespass obstacles.

More specifically, as presented in Fig. 1, a signal that arrives at a receiver might have two types of components: Line of Sight (LOS) and Non-Line of Sight (NLOS). When the signal component is of type LOS, this means that there is a direct path between transmitter and receiver. When the

The authors are with the Wireless Telecommunications Research Group (GTEL), Federal University of Ceará (UFC), Fortaleza, Ceará, Brazil. Diego A. Sousa is also with Federal Institute of Education, Science, and Technology of Ceará (IFCE), Paracuru, Brazil. This work was supported by Ericsson Research, Sweden, and Ericsson Innovation Center, Brazil, under UFC.47 and UFC.49 Technical Cooperation Contracts Ericsson/UFC. Caleb G. Braga was also supported by CAPES.

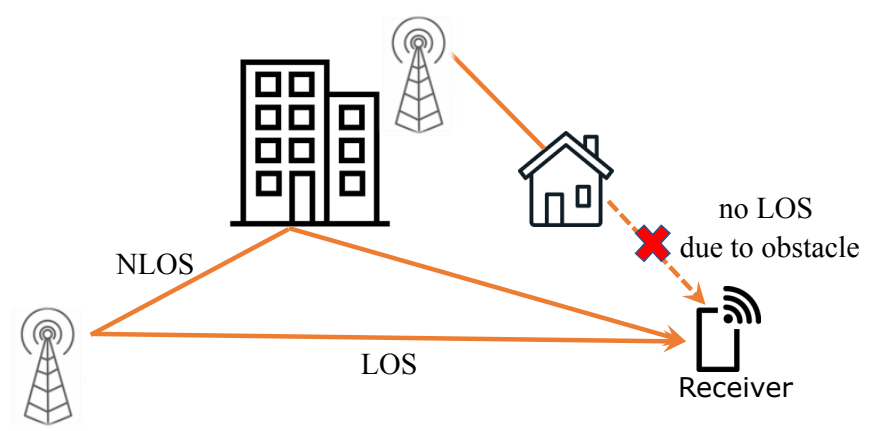

Transmitter

Fig. 1. Representation of LOS and NLOS signals arriving in the receptor.

signal component is of type NLOS, this means that the signal arrives at the receiver after one or more reflections at obstacles in the environment. Since in the mmWave frequencies the propagation loss is high, the NLOS component is weaker than the LOS component. Sometimes, the NLOS component is not even enough to enable the communication between transmitter and receiver. Moreover, as a result of the dynamism of the environment, a signal can loss its LOS component during the communication, e.g., when a car parks between transmitter and receiver. Due to this, it is important to monitor which components exist in the link in order to take actions, if necessary, when there is only the NLOS component, e.g., increase transmission power or choose another link for communication.

To face this challenge, one can model the signal as a Time Series (TS) and try to predict in advance its future quality. Forecasting TS has been the subject of studies for years with many algorithms developed like the statistical model Autoregressive Integrated Moving Average (ARIMA) [2] and the Long Short-Term Memory (LSTM) [3].

Some works have already compared the performance of these algorithms as [4], [5], [6]. In [5], the authors considered the problem of traffic prediction and compared the performance of ARIMA and LSTM. In [6], the authors also compared the performance of LSTM and ARIMA but in other context of TS prediction. However, none of them have specifically focused on LOS/NLOS transitions.

The present paper aims at studying the impact of LOS/NLOS transitions on the signal quality predictions. It is organized as follows. Section II presents the scenario and models adopted in this work. Details of the studied problem and the considered predictor are presented in Section III. The performance of the considered predictor is evaluated via computational simulations in Section IV. Finally, in Section V, 
the main conclusions of this work are summarized and future perspectives are presented.

\section{SYSTEM MODEL}

We considered a downlink Orthogonal Frequency Division Multiple Access (OFDMA) system based on 5G New Radio (NR) specifications [7], in which a User Equipment (UE) was radially moving away from the gNode $\mathrm{B}$ (gNB). The link between the UE and the gNB was modeled as described in [8]. The channel matrix $H$ was defined as a combination of the LOS component, $H^{L O S}$, and the NLOS component, $H^{N L O S}$, as [8]:

$$
H\left(L O S_{\text {soft }}\right)=H^{L O S} L O S_{\text {soft }}+H^{N L O S} \sqrt{1-L O S_{\text {soft }}^{2}},
$$

where the components $H^{L O S}$ and $H^{N L O S}$ were also defined in [8]. $L O S_{\text {soft }}$ was a parameter used to avoid a hard transition in the channel response. It was a floating number between 0 (representing the case with only NLOS) and 1 (representing the case with only LOS) spatially consistent defined as:

$$
L O S_{\text {soft }}=\frac{1}{2}+\frac{1}{\pi} \arctan \left(\sqrt{\frac{20}{\lambda}}(G+F(d))\right),
$$

where $G$ was a spatially consistent Gaussian random variable, $F(d)=\sqrt{2} e r f^{-1}\left(2 \operatorname{Pr}_{L O S}(d)-1\right)$ and $\operatorname{Pr}_{L O S}(d)$ was the distance dependent LOS probability function.

Important to highlight that, from (1), the channel can vary from completely LOS to completely NLOS passing through a moment of transition, when the value of $L O S_{\text {soft }}$ varies from 1 to 0 . Also, from (2), notice that the parameter $L O S_{\text {soft }}$ depends on the distance $d$ between the gNB and the UE. Then, it is expected that the faster the UE moves, the faster the signal transits from LOS to NLOS, i.e., the faster the signal quality deteriorates.

As a measure of signal strength, the UE was measuring the Reference Signal Received Power (RSRP), where the RSRP is the linear average over the power contributions (in Watts) of the resource elements confined within a Synchronization Signal Block (SSB) from the gNB, as defined in [9].

Fig. 2 presents three examples of the time evolution of RSRP measured by a UE radially moving away from the gNB at speed $60 \mathrm{~km} / \mathrm{h}$. The blue curve represents the case where the channel was composed only by a LOS component (LOS $S_{\text {soft }}$ was forced to be equal to 1 ). The orange one represents the case where the channel was composed only by a NLOS component ( $L O S_{\text {soft }}$ was forced to be equal to 0 ). Finally, the green one represents the considered case where the value of $L O S_{\text {soft }}$ was defined as in (2) and the channel transited from LOS only to NLOS only.

\section{ARIMA MODEL}

Considering the channel model described in the previous section and the important decrease in the RSRP value when the channel state transits from LOS to NLOS (as illustrated in Fig. 2), the present work aims at analyzing the impact of this transition in signal quality predictions when the signal

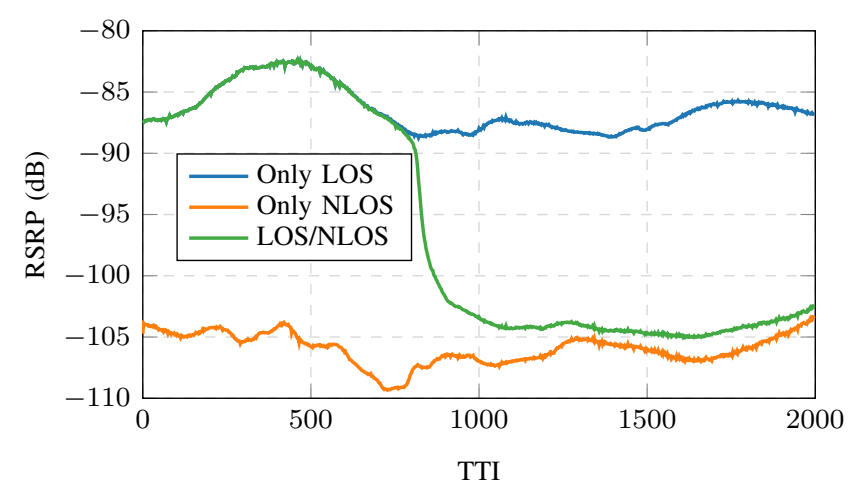

Fig. 2. Examples of RSRP curves measured by a UE radially moving away from the gNB at speed $60 \mathrm{~km} / \mathrm{h}$ in three different scenarios: only LOS (blue), only NLOS (orange) and transition from LOS to NLOS (green).

is modeled as a TS. For this objective, we have selected the ARIMA model, a widely known TS predictor.

ARIMA's main idea is to predict the next sample $x_{t+1}$ of a TS based on previous samples known until instant $t$, according to the following equation [10]:

$$
x_{t+1}=\underbrace{c+\sum_{i=0}^{p-1} \phi_{i} x_{t-i}+w_{t+1}}_{\mathrm{AR}(\mathrm{p})}+\underbrace{\sum_{i=0}^{q} \alpha_{i} w_{t-i}}_{\mathrm{MA}(\mathrm{q})},
$$

where $c$ is a constant, $\phi_{i}$ are autocorrelation coefficients at lags $1,2, \ldots, p, w_{i}$ is a Gaussian white noise series and $\alpha_{i}$ are weights.

The acronym ARIMA is the combination of three other acronyms: AR (autorregressive) + I (integrated) + MA (moving average). Each one of these parts refers to one of the three key elements of the ARIMA model. The influence of AR and MA can be seen in (3). The $\operatorname{AR}(p)$ part is a regression model of order $p$ that explores the dependence between a sample and the last $p$ previous samples. The $\operatorname{MA}(q)$ part is a moving average process that takes into account the dependency between a sample and residual errors. Regarding the Integrated part, $\mathrm{I}(d)$, it allows the ARIMA model to be applied to non-stationary TSs. I $(d)$ corresponds to the $d$-th differentiation of the TS $\left\{x_{t}\right\}$. The purpose of differentiating a non-stationary TS $\left\{x_{t}\right\}$ is to make it stationary [11].

(3) allows the prediction of only the next sample, i.e., $x_{t+1}$, of a TS. In order to predict the next $Y$ samples, i.e., $\left\{x_{i}\right\}_{i=t+1}^{i=t+Y}$, based on the knowledge of the last $X$ samples, i.e., $\left\{x_{i}\right\}_{i=t-(X-1)}^{i=t}$, some implementations of ARIMA uses (3) in an iterative way as follows. First, they predict sample $x_{t+1}$ with (3) based on samples $\left\{x_{i}\right\}_{i=t-(X-1)}^{i=t}$. After, they add the predicted sample $x_{t+1}$ to the set of known samples and predict sample $x_{t+2}$ based on samples $\left\{x_{i}\right\}_{i=t-(X-2)}^{i=t+1}$. This process is repeated until the prediction of sample $x_{t+Y}$.

Next section presents a numerical evaluation of the impact of LOS/NLOS transitions on RSRP predictions using the ARIMA model as predictor.

\section{Performance Evaluation}

Before presenting the simulation results, we present the simulation assumptions. 
TABLE I

SIMULATION PARAMETERS.

\begin{tabular}{ll}
\hline Parameter & Value \\
\hline gNB transmit power & $49 \mathrm{dBm}$ \\
gNB antenna type & URA $8 \times 8$ antennas \\
Antenna element radiation pattern & $3 \mathrm{GPP} 3 \mathrm{D}$ [8] \\
Carrier frequency & $28 \mathrm{GHz}$ \\
System bandwidth & $50 \mathrm{MHz}$ \\
Subcarrier spacing & $60 \mathrm{kHz}$ \\
Number of subcarriers per RB & 12 \\
Number of RBs & 66 \\
Slot duration & $0.25 \mathrm{~ms}$ \\
OFDM symbols per slot & 14 \\
Channel generation procedure & As described in [8]-Fig.7.6.4-1 \\
Path loss & Eqs. UMa in Table 7.4.1-1 of [8] \\
Fast fading & As described in [8]-Sec.7.5 and \\
AWGN power per subcarrier & {$[8]-$ Table7.5-6-UMa } \\
Noise figure & -174 dBm \\
Mobility model & 9 dB \\
\hline
\end{tabular}

\section{A. Simulations Assumption}

A dataset of 600 RSRP TSs was generated according to the scenario defined in Section II, where each RSRP was associated to one realization. The $600 \mathrm{TSs}$ were divided into 3 groups of 200 realizations each: one group with only LOS component during the whole simulation, other with only NLOS component and in the last one the UE transitioned from a state with only LOS to a state with only NLOS according to (1). Each curve of Fig. 2 represents one of these groups. In each realization, the UEs were randomly dropped inside a circle of radius $250 \mathrm{~m}$ with the gNB in the center and they moved away from the gNB in a straight line.

Regarding the usage of the ARIMA model, it was considered that the number of TTIs used as input was equal to the number of TTIs to be predicted (output), i.e., $X=Y$. Four different values were analyzed: 128, 256, 512 and 1024 TTIs.

Concerning the UE mobility, as mentioned in Section II, the time duration of a transition from LOS to NLOS depends on the UE speed, i.e., the faster the UE moves, the faster the transition is. Thus, four different values of UE speed were considered: $30 \mathrm{~km} / \mathrm{h}, 60 \mathrm{~km} / \mathrm{h}, 90 \mathrm{~km} / \mathrm{h}$ and $120 \mathrm{~km} / \mathrm{h}$.

Table I presents other simulation parameters.

\section{B. Numerical Results}

In order to understand how the prediction error evolves in time, firstly, we analyzed, in Figs. 3 and 4, the error prediction time evolution for an specific realization with transition from LOS to NLOS. Figs. 3 and 4 present the impact of speed and prediction size, respectively, on the prediction error time evolution for an specific realization. On the one hand, the time evolution is illustrated at the horizontal axis. On the other hand, the vertical axis on the left hand size of the figures (red curves) represents the RSRP, while the vertical axis on the right hand size of the figures (blue curves) represents the prediction error.

First, for the three figures in Fig. 3, notice that before and after the channel transition we have a low value of prediction

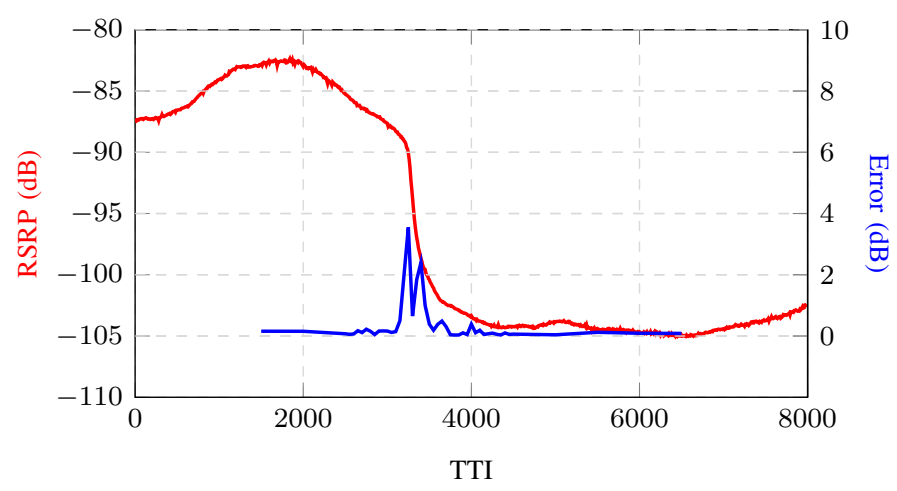

(a) Prediction size $=128$ TTIs.

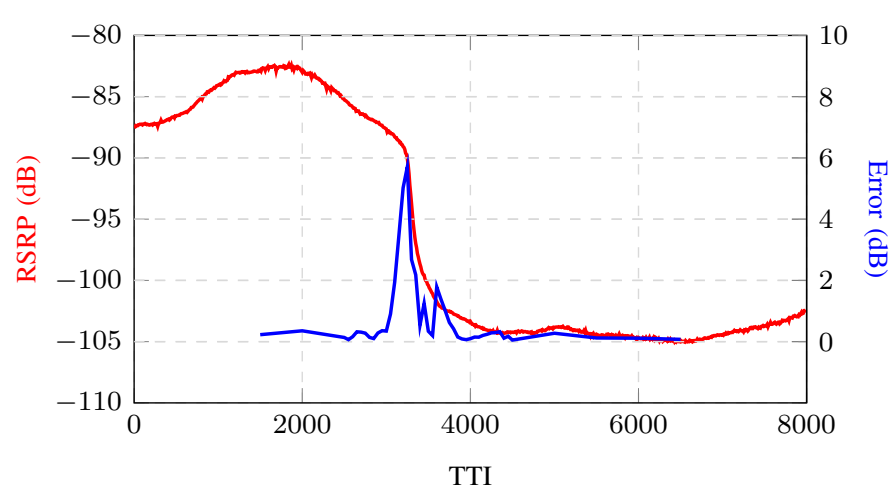

(b) Prediction size $=256$ TTIs .

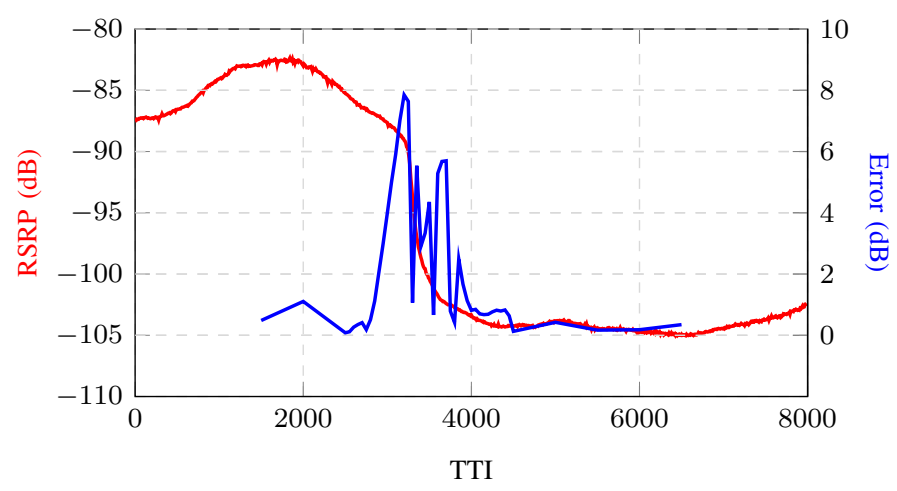

(c) Prediction size $=512$ TTIs.

Fig. 3. Impact of prediction size on the prediction error time evolution for an specific realization $($ speed $=60 \mathrm{~km} / \mathrm{h})$.

error. However, during the channel state transition, the error increases. Also, notice that the two highest peaks of the error curves correspond to the inflection points of the RSRP curves. This is due to the fact that before the inflection point the channel was predominantly in a given state, i.e., LOS, and just after the inflection point the predominant channel state changed, i.e., to NLOS. Thus, at the moment of the occurrence of an inflection point, previous samples of RSRP were highly uncorrelated with future samples inducing a high prediction error. Furthermore, notice that when increasing the prediction size, the prediction error during the channel state transition increases. This can be explained by the fact that, for higher values of prediction size, we try to predict values even further in the future which are even more uncorrelated with the 


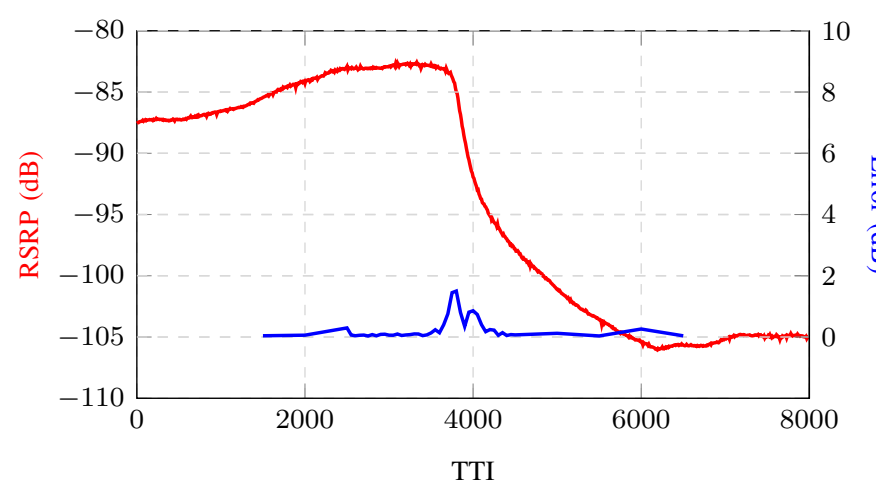

(a) Speed $=30 \mathrm{~km} / \mathrm{h}$.

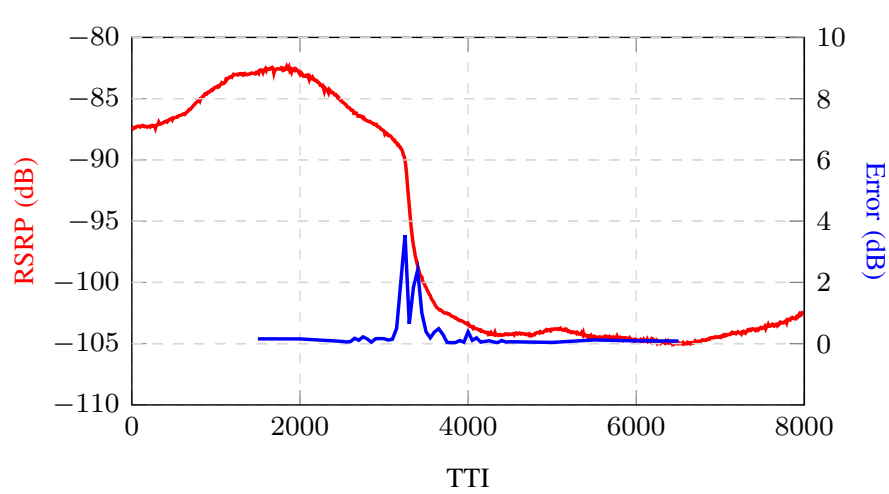

(b) Speed $=60 \mathrm{~km} / \mathrm{h}$.

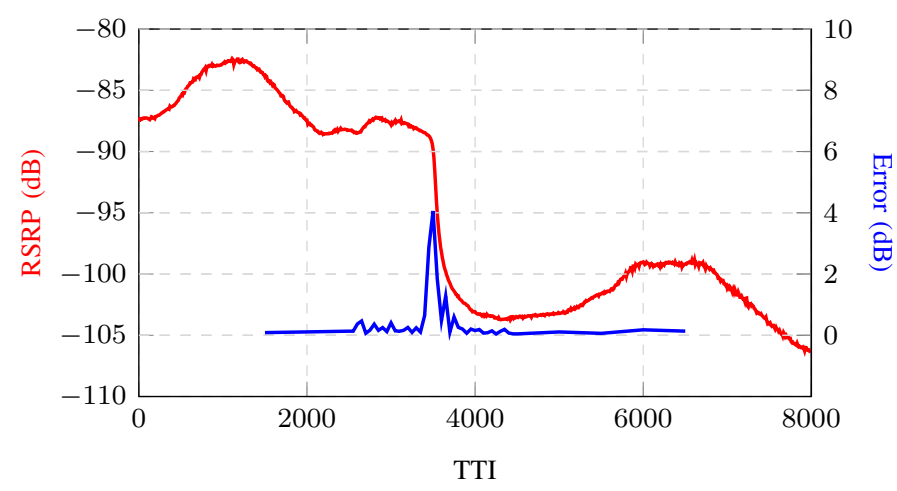

(c) Speed $=90 \mathrm{~km} / \mathrm{h}$.

Fig. 4. Impact of speed on the prediction error time evolution for an specific realization $($ Prediction size $=128$ ).

previous samples.

Regarding the impact of speed on the prediction error illustrated in Fig. 4, analyzes similar to the ones of Fig. 3 can be performed. An interesting point to be highlighted is that when increasing the speed value, the error increases during the channel state transition. This happens because, when moving faster, one moves a higher distance in a given time window. Thus, RSRP samples from one TTI are more uncorrelated to samples of a next TTI.

Figs. 5 and 6 present the impact of speed and prediction size, respectively, on the $90 \%$-ile of the CDF of the prediction error time evolution for the three different groups of simulated channel state. As already mentioned, the three groups are: one with only LOS component during the whole simulation,

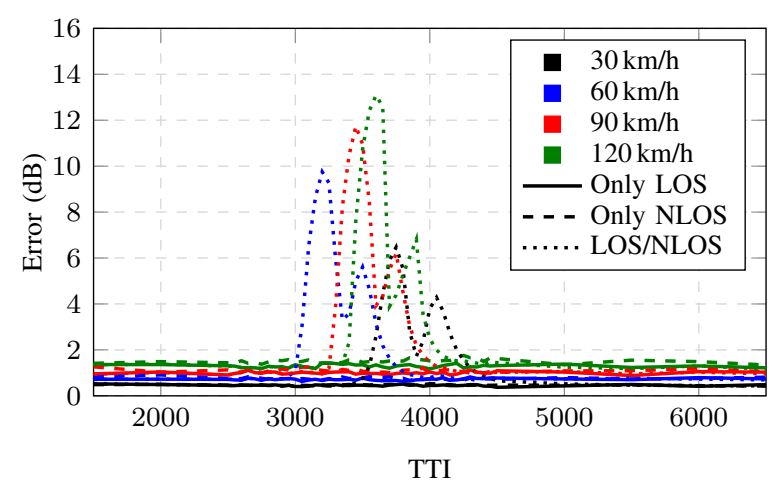

Fig. 5. Impact of speed on the time evolution of the $90 \%$-ile of the CDF of the prediction error, considering prediction size equal to 256 TTIs.

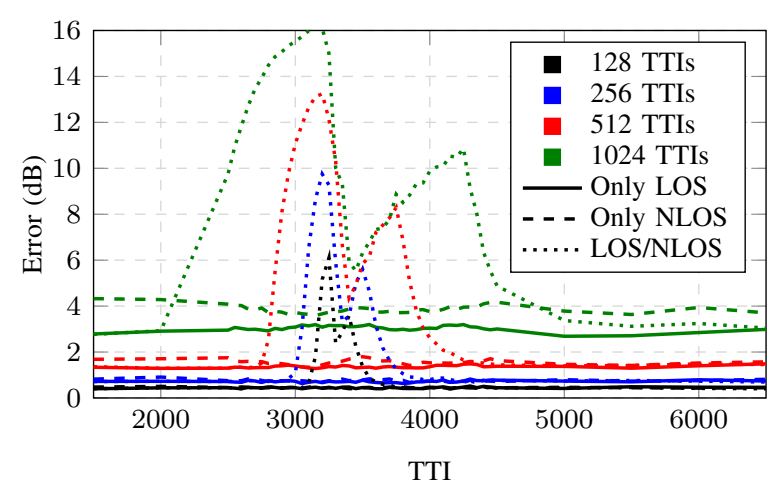

Fig. 6. Impact of prediction size on the time evolution of the $90 \%$-ile of the $\mathrm{CDF}$ of the prediction error time evolution, considering speed equal to $60 \mathrm{~km} / \mathrm{h}$.

other with only NLOS component and in the last one the UE transitioned from a state with only LOS to a state with only NLOS. For each group, we considered 200 realizations and for each time instant we calculated the CDF of the error prediction. Each time instant of Figs. 5 and 6 presents the $90 \%$-ile of the calculated CDF at that TTI.

Concerning Fig. 5, first, on the one hand, we highlight that the groups with only LOS and only NLOS presented a prediction error lower than $2 \mathrm{~dB}$ during the whole simulation. On the other hand, the group with the transition from LOS to NLOS during the simulation presented a high error during the transition. Besides, it is important to remark that, since the speed of each curve is different, the moments at which each curve performed the transition LOS to NLOS was different, that is why the error peaks in Fig. 5 occur in different TTIs.

Comparing Fig. 5 and Fig. 6, we can notice that the error prediction is more sensitive to a variation of the prediction size than a variation of the speed. This happens because during the transition, samples further in the future are more uncorrelated with previous samples.

\section{Conclusion and Future Perspectives}

The present work presented the impact of LOS/NLOS transitions on signal quality predictions when the signal is modeled as a Time Series. It was concluded that during a LOS/NLOS transition, the error increases a lot, achieving 
values that make the predictions almost impracticable to be used. We have showed that two most critical moments are the ones of the LOS/NLOS transition inflection points, since at that moments, previous and future samples become more uncorrelated than at other moments.

In order to enable future networks to take advantage of TS signal quality predictions, the problem of increased prediction error during a LOS/NLOS transition must be addressed. One way is to not take into account only the signal quality itself, but also other information, such as, environmental characteristics. Other possibility which we are currently investigating is to dynamically adapt the predictor characteristics based on current error value.

\section{REFERENCES}

[1] A. Gupta and R. K. Jha, "A Survey of 5G Network: Architecture and Emerging Technologies,"in IEEE Access, vol. 3, pp. 1206-1232, 2015, doi: 10.1109/ACCESS.2015.2461602.

[2] Robert H. Shumwayy and David S. Stoffer:Time Series Analysis and Its Applications. Springer, 2017.

[3] A. Géron, Hands-on Machine Learning with Scikit-Learn, Keras \& TensorFlow. O'Reilly, 2019.

[4] A. Kulkarni, A. Seetharam, A. Ramesh and J. D. Herath, "DeepChannel: Wireless Channel Quality Prediction Using Deep Learning,"in IEEE Transactions on Vehicular Technology, vol. 69, no. 1, pp. 443-456, Jan. 2020, doi: 10.1109/TVT.2019.2949954.

[5] S. Jaffry and S. F. Hasan, "Cellular Traffic Prediction using Recurrent Neural Networks,"2020 IEEE 5th International Symposium on Telecommunication Technologies (ISTT), 2020, pp. 94-98, doi: 10.1109/ISTT50966.2020.9279373.

[6] S. Siami-Namini, N. Tavakoli and A. Siami Namin, "A Comparison of ARIMA and LSTM in Forecasting Time Series,"2018 17th IEEE International Conference on Machine Learning and Applications (ICMLA), 2018, pp. 1394-1401, doi: 10.1109/ICMLA.2018.00227.

[7] 3GPP, "NR; NR and NG-RAN Overall description; Stage 2", 3rd Generation Partnership Project (3GPP), TS 38.300, 2020-09, v.16.3.0. [Online].Available: http://www.3gpp.org/ftp/Specs/html-info/38300.htm (visited on 2021-04-13)

[8] 3GPP, "Study on Channel Model for Frequencies from 0.5 to 100 GHz", 3rd Generation Partnership Project (3GPP), TR 38.901, 2017-09, v.14.2.0. [Online].Available: http://www.3gpp.org/DynaReport/38901.htm (visited on 2021-04$05)$

[9] 3GPP, "NR; Physical Layer Measurements", 3rd Generation Partnership Project (3GPP), TS 38.215, 2018-09, v.15.3.0. [Online].Available: http://www.3gpp.org/DynaReport/38215.htm (visited on 2021-04-05)

[10] Sima Siami Namin and Akbar Siami Namin, Forecasting Economic and Financial Time Series: ARIMA vs. LSTM, 2018. arXiv:1803.06386 [cs.LG].

[11] Paul S. P. Cowpertwait and Andrew V. Metcalfe: Introductory Time Series with $R$.

[12] 3GPP, "Study on evaluation methodology of new Vehicle-to-Everything (V2X) use cases for LTE and NR", 3rd Generation Partnership Project (3GPP), TS 38.885, 2018-12, v.15.2.0. [Online].Available: http://www.3gpp.org/DynaReport/37885.htm (visited on 2021-04-05) 